\title{
Experiencias de Inserción Laboral en Jóvenes Obreros en Azcapotzalco, Ciudad de México
}

\author{
The Experience of Labor Insertion in \\ Young Workers in Azcapotzalco, Mexico City
}

\author{
Gustavo Garabito \\ Universidad de Guanajuato
}

\begin{abstract}
El artículo aborda la relación entre trabajo, familia y espacio en jóvenes obreros mexicanos en sus primeras experiencias de inserción laboral en el contexto de la crisis manufacturera actual. Con una metodología cualitativa, se realizaron entrevistas semiestructuradas a una muestra intencionada de 18 jóvenes obreros de Azcapotzalco (8 mujeres y 10 hombres entre 14 y 29 años de edad) y se analizaron sus narrativas bajo una articulación de los ámbitos que conforman su experiencia de trabajo fabril. Entre los hallazgos más importantes destaca cómo los jóvenes obreros construyen su trayectoria laboral a partir de procesos de transición diferenciados por género. En los varones, la salida de la escuela y la inmediata inserción a la manufactura, el nacimiento del primogénito y la conformación familiar se dan en rituales de paso en los que el trabajo predomina sobre la escuela. En el caso de las mujeres, el ingreso al trabajo fabril es el resultado del nacimiento del primogénito y el consecuente abandono escolar, truncando su aspiración de estudios profesionales.
\end{abstract}

Palabras clave: jóvenes, inserción laboral, experiencia, trabajo, México

\begin{abstract}
The article discusses the relationship between work, family, and youth space in young Mexican workers during their first employment experiences in the context of the current manufacturing crisis. With a qualitative methodology, semistructured interviews were conducted with a purposive sample of 18 young workers from Azcapotzalco ( 8 women and 10 men between 14 and 29 years old), whose narratives were analyzed considering the fields that make up their experience of factory work. Among the most important findings, it highlights how youth workers build their career path based on transition processes differentiated by gender. In males, starting work on a factory immediately after leaving school, the birth of the first child, and forming a family constitute rites of passage where work prevails over school. For women, entering factory work is a result of the birth of the first child and the consequent drop out from school, which truncates their aspiration of pursuing professional studies.
\end{abstract}

Keywords: young, labor insertion, experience, work, Mexico

En el contexto de un desempleo estructural, las posibilidades de inserción en el mercado laboral ofrecen pocas opciones deseables y sí muchas alternativas inciertas en un escenario dominado por un ascenso de la flexibilidad laboral, una creciente precarización del empleo y un incremento del sector productivo informal. El segmento juvenil se ve particularmente afectado por su escasa formación educativa y laboral y por su vulnerabilidad ante una oferta de trabajo predominantemente precaria, con pocas o nulas prestaciones sociales. Ante tal escenario ¿cuáles son los significados y experiencias de trabajo que construyen los jóvenes durante los primeros años de empleo y cómo ellos inciden en sus acciones dentro del mundo laboral?

En México, investigaciones socio-demográficas han abordado la problemática del trabajo juvenil (Horbarth, 2004; Oliveira, 2011). Sin embargo, poco se conoce cómo se construye la experiencia subjetiva del trabajo en los jóvenes. El ingreso por primera vez al trabajo es una México.

Gustavo Garabito Ballesteros, Departamento de Estudios Sociales, Universidad de Guanajuato, sede León,

La correspondencia relativa a este artículo debe ser dirigida a Gustavo Garabito Ballesteros, Departamento de Estudios Sociales, Universidad de Guanajuato, sede León, Blvd. Puente Milenio 1001, Fracción del Predio San Carlos, C.P. 37670, León, Guanajuato, México. E-mail: gustavogarabitob@gmail.com 
de las experiencias biográficas más importantes en la vida de cualquier sujeto. Supone incorporarse a la sociedad, madurar, iniciar un proyecto de autonomía (parcial o total), adquirir destrezas y habilidades, participar colectivamente para un objetivo productivo, institucionalizarse y asumir responsabilidades y derechos.

Lo que se vive en el primer trabajo puede ser fundamental para el desarrollo ulterior de la vida laboral. La construcción significativa que se realiza a partir de la experiencia de trabajo paterna y/o materna (o de los hermanos), así como la ética del trabajo enarbolada por el sistema educativo, pueden chocar abruptamente con la realidad del primer trabajo e irse transformando en el transcurso de las experiencias laborales. Los jóvenes en México viven en un entorno laboral que ofrece muchas oportunidades de empleo, pero la mayoría es precaria, informal y/o flexible. El joven tiene que elegir entre trabajar en la calle, en un restaurante o incluso en una fábrica, pero una opción u otra no representa grandes variaciones en cuanto al ingreso, el tiempo invertido y el desgaste físico y emocional.

El largo proceso de reestructuración productiva iniciado en América Latina y particularmente en México, desde 1982 hasta la fecha, ha producido cambios en la configuración sociotécnica de las industrias, a través de la flexibilidad salarial y numérica, la reorganización del trabajo, los usos de tecnología y las relaciones laborales, y en las relaciones entre empresas y con el Estado (De la Garza, 2001). También ha modificado física y vivencialmente el entorno urbano-industrial, transformando la experiencia del trabajo y las interacciones sociales que son construidas cotidianamente por los sujetos.

La delegación Azcapotzalco, en el noroeste del Distrito Federal en México, ha sido una zona urbano-industrial por excelencia. Aunque ahora su liderazgo en la rama de la manufactura ha decaído de manera importante, no solo por un descenso generalizado del sector y su transformación hacia la maquiladora, sino también por el desarrollo económico de municipios como Tlalnepantla y Naucalpan, aún conserva sus características fabriles que se extienden a las colonias y barrios originalmente destinados para obreros (Nieto, 1997).

El estudio que se reporta abordó la construcción de la experiencia subjetiva del trabajo en un contexto de reestructuración productiva, su impacto en el espacio urbano-industrial y su correlación con otros ámbitos de la vida cotidiana, como la familia y la escuela. En este sentido, se inserta en el debate teórico sobre cómo los sujetos atribuyen significados a sus acciones sociales en contextos parcialmente estructurados (el trabajo, la escuela, la economía, el espacio urbano) y cómo estos, a su vez, impactan en las decisiones, acciones y actos de los sujetos, particularmente en la conformación inicial de trayectorias laborales (Da Rosa, Chalfin, Baasch \& Soares, 2011; Román \& Avendaño, 2002).

En los procesos de creación, objetivación y reconstrucción de significados, los sujetos se reconocen como parte de un grupo, una clase social y una comunidad y generan una visión particular del mundo (visión del mundo que no queda solo como imaginario, sino que orienta sus acciones cotidianas) que los identifica y que les permite expresarse. Este estudio buscó precisamente explorar cómo se objetiva la experiencia obrera en la vida cotidiana a través de prácticas, significaciones y apropiaciones en el marco del entorno inmediato (la familia, el barrio) y del mundo social (la crisis de la manufactura).

\section{Referentes Conceptuales: Vida Cotidiana y Experiencia}

La noción de vida cotidiana, como esfera de los aprendizajes, prácticas e interacciones sociales (productivas y reproductivas), funge como telón de fondo para dirigir la mirada sobre la experiencia del trabajo en una etapa en la que esta actividad - a la par de otros eventos igualmente decisivos - influye de manera importante en la transición hacia la adultez. La apropiación del mundo social, a través de la acumulación de la experiencia y de la interacción intersubjetiva con los otros, se encuentra en un proceso de constante renovación y adecuación 
de los esquemas de comprensión, acción y comunicación dentro de la vida cotidiana en el entorno más inmediato, en este caso, la familia, el trabajo, la escuela y el barrio.

Los individuos van construyendo sus propios "mundos particulares" y decidiendo en ellos en el entramado de la vida cotidiana y en las distintas fases de su vida, de acuerdo a su edad dentro de su continuidad biográfica. La elección de amistades, la pareja o el tipo de trabajo va configurando el universo propio, incrementando o transformando las capacidades y experiencias para actuar en situaciones concretas. Al construir su propia esfera de vida, el sujeto se objetiva a sí mismo y al entorno en el que se desenvuelve. La construcción del entorno inmediato (como radio de acción) está delimitada parcialmente por el contexto socio-histórico y de clase. Así, pues, “(...) en la vida cotidiana, el particular se reproduce a sí mismo y a su mundo (el 'pequeño mundo') directamente y el conjunto de la sociedad (el 'gran mundo') de modo indirecto" (Heller, 1998, p. 27).

Dentro de la tradición fenomenológica existe un debate de los términos vivencia (Erlebnis) y experiencia (Erfahrung), que parecieran sinónimos en la obra de Husserl (Montero, 1987). Una solución la ofrece Schütz (1973), quien distingue vivencia de experiencia, al definir la primera como un devenir constante al cual se puede acudir de manera reflexiva, pero que resulta muy general o vago. En cambio, la experiencia denota el acto (Akt) de aprehensión de significado y es fundamental en la construcción del acervo de conocimiento (Jay, 2009; Schütz, 1973). Esta distinción es importante, porque enfatiza el carácter significativo y trascendental de la experiencia por sobre las vivencias comunes.

Las experiencias y prácticas del sector juvenil en la vida cotidiana se distinguen de las de otros grupos etarios, por la relevancia de los procesos de socialización secundaria e inserción social a través de las transiciones hacia la adultez. Así, ámbitos específicos de la vida cotidiana, como el trabajo y la familia, son experiencias fundamentales durante la juventud y su paso a la vida adulta (Pérez, 2010; Saraví, 2009). Entender así las experiencias de transición en los jóvenes implica destacar la heterogeneidad de las distintas dinámicas hacia la adultez, marcadas por el género, condiciones de clase, acceso a la educación y al diverso mercado laboral, entorno espacial y desarrollo de la propia biografía.

Las transiciones de la escuela al trabajo tienen distintas modalidades según sea la salida del ámbito educativo y el ingreso al mundo de trabajo (García, Marino \& Casal, 2006). En los jóvenes obreros de Azcapotzalco entrevistados, la transición de la escuela al trabajo se desarrolló fundamentalmente en transiciones anticipadas y simultáneas, es decir, inmediatamente después de terminar su educación secundaria o media superior — por problemas de disciplina, reprobación de materias o falta de recursos económicos- se insertan en las actividades productivas o bien participan simultáneamente en el trabajo y la escuela.

Esta deserción escolar por el ingreso al mercado laboral, sin duda, es un problema muy serio para la juventud en México. Aun cuando los jóvenes cuentan con una mayor educación que las generaciones pasadas, con un promedio de 10 años de escolaridad, un considerable porcentaje (38\%) de la población juvenil solo termina la educación secundaria (Consejo Nacional de Población, 2010). Según datos de la Encuesta Nacional de la Juventud, a nivel nacional un 43\% de los jóvenes encuestados en 2005 tuvo que dejar de estudiar para incorporarse al mercado laboral, aumentando dicho porcentaje al 54\% entre los hombres de 15 a 19 años, abandonando sus estudios justamente después de haber concluido la secundaria o el primer año de preparatoria (Instituto Mexicano de la Juventud, 2005).

La deserción escolar es particularmente grave en los contextos familiares en situación de pobreza, pues la oferta de trabajo con una preparación escolar de secundaria generalmente tiene condiciones de alta precariedad laboral que no permiten romper los ciclos de pobreza ni disminuir los riesgos de exclusión social. Estas condiciones de reproducción de la pobreza, salida prematura de la escuela y segregación de un mercado laboral predominantemente precario 
favorece la condición de jóvenes trabajadores pobres, es decir, un trabajador que junto con su familia no supera el umbral de la pobreza (Organización Internacional del Trabajo [OIT], 2006).

La estructura familiar y de clase parecieran influir en la valoración positiva del trabajo por sobre la educación, pues responden a estrategias económicas de sobrevivencia, según las cuales más miembros de la familia deben incorporarse al mercado de trabajo para complementar los gastos personales y del hogar (Guzmán \& Saucedo, 2007; Saraví, 2009). Por otra parte, en la transición de la escuela al trabajo hay componentes de género que diferencian la experiencia de estos cambios entre hombres y mujeres. En el caso de los varones, ello se puede entender como parte de los "ritos tradicionales de paso" hacia la adultez en la construcción de la masculinidad, en la que la pertenencia al ámbito de la producción es fundamental para desarrollar los roles de trabajador y proveedor del hogar, en suma, del "ser hombre". Esto contrasta con el imaginario tradicional de la construcción social de la mujer, quien, mientras no sea marginada al ámbito reproductivo en las labores domésticas y el cuidado de los hijos, puede continuar con sus estudios (Pérez \& Urteaga, 2001; Vendrell, 2002).

En ese sentido, las mujeres jóvenes se ven obligadas a renunciar a sus estudios de preparatoria por las necesidades económicas que priman en su hogar y que no pueden eludir, por unirse o casarse o por el nacimiento de su primogénito, sucesos — sobre todo los últimos dos- estrechamente ligados entre sí, como se podrá ver más adelante. De esta manera, por ejemplo, en el año 2005 solo un tercio de las mujeres de 19 años acudía a la escuela (Colín \& Villagómez, 2010). Esta distinción entre la valoración escolar desde el punto de vista del género y la ruptura en su trayectoria educativa es crucial para comprender las grandes diferencias entre la experiencia obrera de los varones y las mujeres.

Esta investigación reconstruyóla experiencia del trabajo obrero de los jóvenes de Azcapotzalco y su relación con la vida cotidiana, a partir de la articulación horizontal de narrativas en torno a la inserción laboral, las condiciones laborales y las experiencias de trabajo. El foco estuvo puesto en el proceso de transición entre escuela y trabajo y, en el caso de las mujeres, en la maternidad. La pregunta de investigación se refiere a las condiciones bajo las cuales se da el paso de la escuela al trabajo manufacturero, es decir, cómo es que se elige el empleo fabril como primer trabajo entre los jóvenes de Azcapotzalco.

\section{Método}

\section{Diseño}

El análisis de las trayectorias y experiencias de trabajo de los jóvenes obreros de Azcapotzalco requirió de una articulación multidimensional de distintas categorías que tienen como columna vertebral la actividad productiva. Dado que el foco central son los procesos subjetivos de dotación de sentido y significados, se optó por una estrategia metodológica cualitativa, la que resulta adecuada para la recuperación, análisis y comprensión de este tipo de fenómenos (ÁlvarezGayou, 2005; Ruiz, 1996).

\section{Participantes}

En el estudio participaron 18 jóvenes obreros (8 mujeres y 10 hombres), residentes en la delegación Azcapotzalco, que habían trabajado en la manufactura en los polígonos industriales de dicha delegación. Se diseñó un muestreo intencionado, utilizando los siguientes criterios de selección: que vivieran en Azcapotzalco desde su nacimiento, que solicitaran empleo como ayudante general en la manufactura, que hubieran laborado en dicho sector en polígonos industriales de la delegación y que su edad oscilara entre los 14 y 29 años. 
Para seleccionar a estos jóvenes se consultaron bases de datos de la bolsa de trabajo de la delegación, correspondientes a enero y febrero de 2009 y, vía telefónica, se les solicitó tener una reunión para realizar la entrevista presencial e individualmente, previa solicitud formal a través de una carta institucional de parte de la Universidad Autónoma Metropolitana.

Se realizaron alrededor de 108 llamadas, pero en aproximadamente 52 llamadas el número estaba fuera de servicio, era incorrecto o la persona ya no vivía en ese domicilio. Asimismo, en cerca de 38 casos, si bien sí se contactó a la persona, nunca se logró un encuentro cara a cara para la realización de la entrevista, ya sea por falta de tiempo del joven obrero o por su negativa a participar. Un participante que no cumplía con los criterios de selección (un padre de dos jóvenes trabajadores) también fue entrevistado por la relevancia de su narrativa.

\section{Instrumentos}

Se aplicaron dos instrumentos: (a) observación no participante de las características físicas de los barrios, vecindades y los entornos industriales de la delegación; y (b) entrevistas semiestructuradas de preguntas abiertas que fueron realizadas a los jóvenes obreros bajo las temáticas de: (i) condiciones de trabajo y trayectoria laboral, (ii) trabajo y familia, (iii) escuela y (iv) vivencias y recuerdos de las colonias y barrios de Azcapotzalco.

\section{Procedimiento}

La recolección de información y narrativas que aquí se exponen se dio en dos fases. En la primera etapa, de tipo exploratorio, se realizó observación no participante durante tres semanas, recorriendo las zonas industriales y barrios obreros de la delegación Azcapotzalco. Con ella se pudieron conocer las dinámicas cotidianas entre los espacios urbanos y los industriales. En la segunda etapa, luego de obtener oralmente el consentimiento de los jóvenes obreros para participar en el estudio, el autor de este artículo realizó las entrevistas semiestructuradas a estos en su hogar o espacio de trabajo. Las entrevistas tuvieron una duración promedio de dos horas, fueron grabadas y posteriormente transcritas para el proceso de análisis.

\section{Análisis de la Información}

Las narrativas obtenidas en las entrevistas fueron sometidas a un análisis cruzado en función de las categorías de interés del estudio, es decir: trabajo, familia, escuela y espacio. Bajo la perspectiva del construccionismo social (Berger \& Luckmann, 1997; Gergen, 1996), se realizó una matriz de análisis donde se articulan los conceptos ordenadores más generales de jóvenes, trabajo y experiencia con las categorías analíticas eje: escuela, familia y espacio (barrio). Las narrativas de los jóvenes obreros fueron sistematizadas y reconstruidas a partir de dicha matriz en un juego de convergencias y divergencias entre los distintos testimonios.

\section{Resultados}

La presentación de los resultados se desarrolla a partir de las categorías eje que guiaron el análisis de las narrativas de los jóvenes trabajadores entrevistados, la experiencia de inserción laboral en el marco de: (a) las trayectorias escolares truncadas, (b) las exigencias económicas familiares y (c) el entorno industrial urbano. Para proteger la identidad de los entrevistados los nombres que aparecen al final de cada testimonio que se presenta a continuación son ficticios. 


\section{La Experiencia de Inserción Laboral en el Marco de Trayectorias Escolares Truncadas}

En México, la salida anticipada del sistema educativo y el inmediato ingreso al mercado laboral responde a distintas dinámicas familiares y económicas. En parte, la inserción laboral inmediata tiene lugar porque el paso de la secundaria al nivel medio superior (bachillerato o preparatoria) se ve truncado por los pocos lugares disponibles en las universidades públicas y por no tener la familia recursos suficientes para pagar una escuela privada.

El tiempo ocioso, resultado de no estudiar ni trabajar, genera en estos jóvenes narrativas de enfado y aburrimiento y propicia presiones de la familia para que se empleen, para que "sean útiles" y aporten económicamente, por lo menos, con los propios gastos del joven, lo cual es visto por ellos como una buena oportunidad para obtener ingresos propios. Son principalmente los varones entrevistados quienes ingresaron a su primer trabajo justo después de terminar la secundaria, sin mostrar interés por continuar sus estudios hacia la preparatoria o el bachillerato de manera continua.

El tipo de actividades que desempeñan los jóvenes obreros, como ayudantes generales, implica un horario de al menos ocho horas diarias y actividades físicamente muy desgastantes. Este tipo de empleo, a diferencia de otros en el sector comercio o servicios, deja poco margen para que estos trabajadores puedan continuar sus estudios y mucho menos para que desempeñen ambas actividades de manera simultánea. En cambio, las mujeres fueron las que más se esforzaron por desempeñarse simultáneamente en el trabajo y el estudio, aunque sin mucho éxito.

[¿Cómo fue que comenzaste a trabajar?]

Porque no estudiaba... y, pues, me obligaron a trabajar... ya que no estudio, pues trabajo. (Luis, 19 años)

[¿Cómo fue que entraste?]

Pues, como no tenía nada que hacer [en el lapso de la secundaria y la preparatoria], me fui a dar unas vueltas y vi ahí [un letrero que decía] "se solicita ayudante general". Fui, toqué y me dijeron que sí. (Miguel, 21 años)

En la visión en torno a la escuela y el trabajo destaca una importante distinción por género, pues para los varones la escuela no es una gran prioridad, en contraste con lo que sucede entre las mujeres. En las narrativas de los jóvenes varones se puede apreciar cómo la inmediata transición al trabajo después de la secundaria se toma como un paso a seguir casi de manera automática y natural, pues el trabajo, dentro del imaginario social, es considerado un ámbito predominantemente masculino.

Además de comenzar a ejercer el rol de trabajador como parte de la construcción de la masculinidad, se debe considerar que el empleo de ayudante general en la manufactura implica un considerable esfuerzo físico, generalmente relacionado con lo masculino: cargar, limpiar maquinaria, cortar, apilar cajas, entre otros. Adicionalmente, el joven trabajador tiene que tolerar humillaciones y bromas diversas de connotación sexual de sus compañeros de mayor edad y antigüedad en el trabajo, con el fin de ganarse su respeto y formar parte del grupo de trabajo. De esta manera, el trabajo no funge solo como el inicio de una etapa productiva, sino que también es parte esencial en los procesos de socialización dentro de los marcos significativos de la masculinidad tradicional y del ser un trabajador. Además, estos jóvenes perciben el término de la educación secundaria como el fin de una etapa de "desmadre", es decir, de consumo de alcohol y drogas y, en algunos casos, de pertenencia a pandillas. Conciben y asumen su nueva etapa como trabajadores — con o sin la preparatoria - como un episodio para madurar y prepararse para una vida productiva.

La preponderancia del ámbito laboral por sobre el educativo en los jóvenes obreros (en algunas obreras también) se puede explicar por lo significativo de una vivencia sobre la otra. Como se verá más adelante, el primer trabajo para los jóvenes obreros de Azcapotzalco es una 
experiencia mucho más emocionante e interesante que su permanencia en los estudios, pues la actividad laboral implica la inclusión no solo en la esfera de la productividad, sino también en el mundo del consumo, además de que les permite lograr una relativa independencia. En contraste, continuar con los estudios — si es que se consigue un lugar en la preparatoria- es prolongar un estatus de dependencia con los padres o, en todo caso, limitar su actividad laboral a empleos de medio tiempo. Para las jóvenes trabajadoras esta transición es mucho menos tajante, pues, en contraste con sus pares varones, la mayoría de las obreras entrevistadas inició sus estudios de preparatoria y su salida se debió fundamentalmente al nacimiento de su primogénito o a la reprobación e incumplimiento de las materias. En el caso de aquellas jóvenes trabajadoras que ni siquiera iniciaron sus estudios medios superiores, ello se debió a la necesidad de trabajar, al tener a sus hijos a edades tempranas.

En ese sentido, los varones toman la elección de laborar en vez de continuar con sus estudios y las mujeres se ven obligadas a dejar sus estudios y comenzar a trabajar. En el mismo tenor, la circunscripción del trabajo manufacturero a esquemas simbólicos y significativos masculinos podría sugerir el hecho de que ninguna de las jóvenes entrevistadas inició su vida laboral en una fábrica, sino más bien en el sector de comercio o servicios. La opción fabril para las mujeres es resultado de un agotamiento de otras opciones laborales deseadas, lo cual explica los sentimientos de frustración que constantemente compartieron las obreras.

Destaca el caso del único adulto entrevistado, un taxista de trayectoria obrera con un hijo obrero y una hija profesional (diseñadora gráfica), quien ilustra las dos trayectorias laborales de sus hijos y las diferencias en la experiencia de trabajo:

Los dos se van juntos en la mañana a trabajar allá a Vallejo, pero pues mi hijo no quiso seguir estudiando $y$ ahí anda de obrero, como yo, pero mi hija sí aprovechó y estudió y ahí anda diseñando tenis. Y aunque trabajen muy cerquita uno del otro, pues no es lo mismo andar de obrero que ya tener tu profesión.

[¿Y cómo los ve a los dos?]

Aunque parezca mentira, veo más contento, ahora sí que como que más relajado a mi hijo en lo que hace, que a mi hija, toda presionada y trabajándole duro. Pero a la hora de la paga, pues ahí sí se nota la diferencia.

(Oscar, 42 años)

En este tránsito educativo que se trunca al término de la educación secundaria o a la mitad de preparatoria, la percepción que se tiene de la escuela es sumamente relevante para explicar estas transiciones hacia el trabajo. Se encuentra una valoración contradictoria de la educación, pues, por una parte, los jóvenes obreros de Azcapotzalco saben que la escolaridad no garantiza buenos empleos, ya que conviven con trabajadores con mayor escolaridad pero en los mismos puestos de trabajo, pero, al mismo tiempo, anhelan obtener una mayor educación, por lo menos terminar la preparatoria y cursar alguna carrera técnica o incluso profesional.

Los estudios medios superiores son vistos solo como un trámite para alcanzar posiblemente un mejor puesto, no como la adquisición de nuevas habilidades para el desempeño laboral o la continuidad de estudios universitarios. A diferencia de otros sectores juveniles, para los jóvenes obreros una carrera universitaria no forma parte de su plan de trayectoria educativa:

Ahora si que los estudios ya no importa, eh, porque yo he conocido personas con estudios y son obreros. (Vanessa, 27 años)

[¿Crees que elegiste bien el momento de trabajar?]

Me hubiera esperado un poco más, me hubiera puesto a estudiar, porque luego el estudio te da más... bueno, a veces te da más, pero luego ya no, luego tienen carreras y no les sirven de más... un compañero acabó su carrera y está trabajando en un café.

[¿Piensas estudiar más adelante?]

Quiero estudiar, acabar por lo menos la prepa, porque un tío me va a meter a Cable Visión, pero si acabo la prepa, y quiero trabajar ahí. (Luis, 19 años) 
Esta aparente contradicción respecto de la valoración de la escuela responde a su propia experiencia de trabajo, pues en sus primeros empleos, al aprender nuevos conocimientos prácticos vinculados con su actividad, subestiman la validez de seguir con su trayectoria escolar; pero con el paso del tiempo, la imposibilidad para ascender a otros puestos u otros empleos mejor remunerados se la adjudican a su escasa educación. En las zonas industriales de Vallejo y San Antonio destacaba la presencia de varios profesionales (contadores, administradores y psicólogos en recursos humanos) que, si bien pretendían obtener puestos lo más cercanos a su perfil profesional, estaban en la disposición de emplearse en lo que encontraran.

En la construcción de significados en torno a la escuela y el trabajo, el ámbito laboral adquiere mayor relevancia en los jóvenes obreros varones de Azcapotzalco, pues adquieren distintas habilidades específicas que van conformando su perfil de trabajo: el manejo de ciertas máquinas (e.g., cortadoras, troqueladoras, hornos), el manejo de vidrios, aprender a conducir, a estibar, entre otros; y otros conocimientos tácitos no tan técnicos, pero igualmente importantes: saber administrar su remuneración semanal, vales de despensa y sus documentos personales, levantarse temprano y desenvolverse con sus compañeros y superiores.

[¿Y cómo te fue en la vidriera?]

[Emocionado] No, pues subí de ayudante general, después a aprendiz y después de aprendiz A, aprendiz AA

$y$ hasta operador de horno.

[¿Qué tanto aprendiste en todo ese tiempo?]

Pues, ahora sí que aprendí el oficio del vidrio, aprendí a cortar, a templar... ahorita ya se me fue la onda del proceso del vidrio puro. (Erick, 25 años)

La incorporación de estas destrezas es mucho más vivencial y tiene resultados inmediatos en su entorno laboral y económico. Además, en tanto que son hijos de obreros, los anhelos de horizontes profesionales son casi nulos o son vistos como muy lejanos y en función de obtener un mejor puesto y remuneración, no de una carrera específica. Una obrera de Vallejo comentaba que le gustaría ser licenciada y, a la pregunta sobre qué le gustaría estudiar, respondió que no sabía, que solo quería ser licenciada.

\section{La Experiencia de Inserción Laboral en el Marco de Exigencias Económicas}

Las estrategias económicas familiares, además de una dimensión fáctica, es decir, la exigencia de trabajar para aportar ingresos al hogar, tienen una dimensión simbólica, pues los procesos de significación en torno al trabajo se construyen desde las concepciones que han desarrollado los padres y, en menor medida, los hermanos trabajadores mayores. Los significados en torno al trabajo tienen un componente ideal, pero otro práctico en función del empleo que se anhela y del que existe, que está disponible y satisface las necesidades más urgentes.

Los jóvenes de padres obreros o con oficios (albañil, cerrajero, soldador) y en situación de pobreza tienen una visión del trabajo generalmente pragmática e inmediata y está en función de la obtención de ingresos y no necesariamente de una realización personal o profesional, como podría ser el caso de un joven de clase media, hijo de profesionales. Esta concepción pragmática del trabajo es transmitida cotidianamente a los hijos cuando optan por su primer empleo, lo cual acelera su proceso de inserción laboral en detrimento de su desarrollo educativo. Es importante advertir que, si bien hay casos en los que los padres desean que sus hijos continúen con sus estudios, la posibilidad de que esto ocurra está en función de la economía del hogar, pues la educación por lo general se puede postergar hasta que las condiciones lo permitan.

La construcción de significados del trabajo no debe entenderse como impuesta o transmitida a manera de enseñanza, sino que es reconstruida por los jóvenes a partir de las vivencias, discursos y prácticas que los padres y hermanos desarrollan en torno al trabajo: 
[¿Cómo decidiste entrar?]

Pues, más que nada fue por necesidad... porque sí mi padre trabajaba, pero ganaba muy poquito; mi mamá también estaba trabajando, mis hermanos también estaban trabajando, pero, pues, ahora sí que cada quien para sus gastos, porque era poca la paga.

[¿Necesidades para tu familia o para las tuyas propias?]

En parte sí, propias, porque, pues, decía "si ahorita no estoy estudiando, pues, de perdida me pongo a trabajar, a hacer algo de provecho" y ya me metí a trabajar y, pues si, sí le daba a mi mamá para el gasto y lo que quedaba, pues, era para mis cosas personales. (Alberto, 29 años)

Los obreros entrevistados rara vez logran trabajar y estudiar simultáneamente, porque no tienen los recursos para seguir estudiando y/o no cuentan con el tiempo suficiente para las tareas escolares, y no es posible sostener ambas actividades, generándose así un círculo vicioso, pues, al no poder continuar con sus estudios al nivel medio superior, quedan constreñidos a un mercado laboral que no genera ni los ingresos suficientes ni las condiciones para seguir estudiando:

[¿No has buscado otro trabajo que no sea en fábrica?]

No, más bien, por lo mismo que no tengo muchos estudios, ahora sí que es donde encuentre. Una vez quise buscar un trabajo de recepcionista. Dije "voy a cambiar, a lo mejor me va mejor”, pero pedian computación y, pues, yo no sé nada y, pues, perdí ese trabajo... y, pues, las escuelas de computación, pues, es pagar y, pues, es otro obstáculo y perdí esa oportunidad, tenía todo, menos la computación... ahorita lo que sí quiero es seguir estudiando la prepa abierta y, por lo mismo, estoy buscando un trabajo que me permita hacerla los sábados. (Vanessa, 27 años)

Destaca que los jóvenes que abandonaron sus estudios para trabajar y así aportar al ingreso familiar provienen de hogares de padres obreros que también trabajaron en zonas industriales cercanas (Vallejo, San Antonio, Santa Inés, Santo Domingo) y que requieren del trabajo de toda la familia para solventar los gastos domésticos. Y, en ese sentido, los hijos obreros, al insertarse en contextos igualmente precarios que el de sus padres, contribuyen a mantener condiciones de exclusión social que los imposibilita para mejorar sus niveles de vida. Constituyen familias que la Organización Internacional del Trabajo ha llamado trabajadores pobres (OIT, 2006), en las que, aun cuando la mayoría de los miembros de la familia labora, ello no es suficiente para ascender de estrato socioeconómico.

Resulta interesante que, aun cuando la diversidad del empleo informal se erige como una opción atractiva que permite administrar con relativa libertad el tiempo, permitiéndoles estudiar de manera simultánea y con ingresos ligeramente superiores a las ofertas de empleo tradicionales en la manufactura y el comercio establecido, es justamente la tradición familiar de carácter obrero, las redes sociales (familiares y vecinales), la inmediación del espacio fabril y la búsqueda de un mínimo de seguridad social — que no ofrece el sector informal— lo que orienta a estos jóvenes trabajadores de Azcapotzalco a buscar empleos en la manufactura por sobre otras opciones del mercado laboral. Incluso, algunos obreros entrevistados informalmente en Vallejo daban cuenta de trayectorias predominantemente manufactureras, pero con breves episodios en el comercio informal como ayudantes — no destinaban capital para la compra de mercancía-, episodios que narraban con descontento.

La edad de los jóvenes también mostró algunas diferencias. Así, los jóvenes mayores (25-29 años) son los más preocupados por la tensión que les produce seguir estudiando para aspirar a mejores empleos, al mismo tiempo de cumplir con sus responsabilidades laborales. En los muchachos más jóvenes (16-21 años) esta tensión no está presente: la escuela es desplazada por el trabajo de manera indefinida y, si bien tienen proyectado seguir estudiando, no fijan plazos ni planes para hacerlo. Una primera interpretación de esta diferencia podría estar en torno al deterioro físico que los jóvenes de mayor trayectoria obrera sufren dentro del sector manufacturero, y al desgaste emocional, dado los estrechos límites que ofrece el sector en términos de aumentos salariales, ascenso a otras jerarquías o mejores puestos. Son ellos quienes más desean 
cambiar su condición obrera, pero para ello requieren un mayor nivel educativo. En cambio, los obreros más jóvenes apenas inician su trayectoria laboral, la cual viven como novedosa e interesante y no se preocupan demasiado por sus horizontes laborales ni educativos.

\section{La Experiencia de Inserción Laboral en el Marco del Entorno Industrial Urbano}

También influye de manera notable en la inserción laboral de los jóvenes en el sector manufacturero el entorno industrial urbano, no solo por la cercanía de la oferta laboral, sino también por las imágenes y los significados creados desde la infancia, al convivir en un ambiente con una importante presencia de fábricas. Esta influencia también se presenta en las redes sociales que facilitan la inserción al trabajo, pues, al estar en un mismo espacio, las referencias al trabajo fabril se refuerzan por medio de amigos y familiares que ya laboran en las zonas industriales cercanas o en las fábricas diseminadas entre las colonias y barrios de Azcapotzalco. De alguna manera las redes sociales dotan de información para acceder a este mercado de trabajo fabril, el cual, específicamente en las zonas industriales de la demarcación, da prioridad a la cercanía en la selección del personal.

Destaca el uso del espacio, vivido como un recurso en la elección del trabajo: la cercanía con el hogar, el ahorro en el transporte público, la invitación y convivencia en la fábrica con vecinos, amigos y familiares - lo cual facilita la experiencia del primer trabajo - y el ahorro en tiempo al buscar el empleo, pues las vacantes ofertadas son visibles afuera de las fábricas y talleres de las zonas industriales cercanas (Vallejo, Santa Inés, Santo Domingo) o dentro de las mismas colonias y barrios. Estas ofertas se comunican de manera muy rápida entre los vecinos y amigos, además de estar disponibles cotidianamente en los trayectos diarios. Es decir, vivir en Azcapotzalco, aun con la franca decadencia de la industria manufacturera en la zona, es vivir en un entorno con una importante significación espacial de trabajo, la que es experimentada no solo por los habitantes de la delegación, sino también por los forasteros.

Estas características espaciales permiten que las fases de desempleo sean una cuestión de elección propia "para descansar", dada la relativa abundancia de empleo, por lo menos para las vacantes de ayudante general. Asimismo, esta situación espacial podría explicar la recurrencia de trayectorias laborales más o menos homogéneas, distribuidas en un radio geográfico reducido al interior de la delegación Azcapotzalco. Por otra parte, resulta muy interesante que muchas de las significaciones que se anuncian desde la descripción del primer trabajo tienen una presencia en todas las narraciones individuales:

[¿Tu primera idea era trabajar en la fábrica?]

Pues no, pero no me quedaba de otra.

[¿Y nunca pensaste en algún ciber café o algo así?]

No, porque son muchas horas y aqui solo son nueve horas de lunes a viernes y el pago eran $\$ 750$ semanales

(...) y porque estaba bien cerca y en los demás trabajos, pues, tenía que caminar o gastar en pasajes y en este podía venir a comer y luego ya me iba caminando, hacía como unos tres minutos. (Luis, 19 años)

Como se puede apreciar, el entorno industrial urbano es una pieza clave en la búsqueda de empleo por parte de estos jóvenes. Hay una asociación entre las condiciones de su deserción escolar, la trayectoria laboral de los padres y hermanos y la oferta laboral que predomina en la inmediatez espacial. El espacio articula de manera importante las redes sociales que conducen al conocimiento e ingreso al mercado de trabajo local, pero también les confiere a los jóvenes un sentido de familiaridad respecto de la elección natural del empleo que se busca. Así, el trabajo es parte natural del paisaje y de la vida cotidiana, mucho antes de que se comience a experimentarlo. 


\section{Discusión}

La articulación del espacio, las necesidades personales y familiares y la situación escolar convergen notoriamente en la elección del trabajo fabril. Si bien no todos los jóvenes experimentaron el trabajo manufacturero como su primer empleo, ciertos sucesos vinculados a la educación y a la maternidad/paternidad, y la preeminencia de un entorno fabril -aunque decadente- hacen que este tipo de trabajo se imponga sobre otras posibilidades laborales. Aun cuando los motivos y necesidades de trabajo varían entre los jóvenes entrevistados, el uso del espacio y la familia, como recursos para emplearse por primera vez, se mantiene como una constante en las experiencias laborales aquí vertidas. El abandono escolar, sobre todo al término de la secundaria, ya sea por desinterés en seguir estudiando o por la carencia económica familiar, en términos generales, uno de los principales motivos de inserción laboral. De igual forma, el inicio de la maternidad/paternidad temprana sería la razón para insertarse en el trabajo fabril.

Esta incapacidad del ámbito escolar para garantizar satisfacción en el estudio y un futuro laboral deseable ha sido llamada la escuela acotada (Saraví, 2009), jugando la clase social un papel determinante en este fenómeno, pues en las clases populares la importancia de la educación formal disminuye ante la formación para el trabajo, porque, además de obtener un aprendizaje aplicado para su puesto de trabajo, obtienen ingresos que no podrían tener si solo estudiaran.

Además, otras circunstancias, tales como la jornada escolar (matutina/vespertina), influyen en la inserción laboral, pues bajo el imaginario de que la mayor actividad en una jornada diaria se da durante la mañana, la escuela queda relegada a una fase avanzada del día, en condiciones de mayor desgaste físico y mental de los jóvenes, y donde la probabilidad de insertarse en un empleo es mayor si se estudia en la tarde. En este sentido, la experiencia escolar otorga un status que distingue a los jóvenes trabajadores de aquellos que pueden dedicarse exclusivamente al estudio.

La visión del trabajo y la escuela entre los jóvenes obreros de Azcapotzalco contrasta con la visión de los muchachos que laboran en empleos desarrollados específicamente para el sector juvenil (e.g., en empresas de comida rápida, cadenas de cines), para los que el trabajo es visto como un medio para solventar los estudios y terminar una carrera profesional. A pesar de lo difícil que es para estos jóvenes cumplir con una jornada escolar y otra laboral, esta última queda subordinada a la primera, en oposición con los jóvenes obreros, para los que el trabajo reemplaza a la escuela, total y permanentemente (Garabito, 2009).

En el caso de los jóvenes obreros de la delegación Azcapotzalco, la transición escuela-trabajo está estrechamente supeditada más a las condiciones materiales que prevalecen en el hogar y a las prácticas y discursos en torno al trabajo por parte de los padres que al nivel educativo. Aun cuando la escolaridad los margina a un mercado laboral predominantemente precario y con bajos salarios, no es el detonante exclusivo para su inserción en actividades productivas manufactureras. Su inserción laboral responde a una visión inmediata y pragmática del trabajo, que acelera sus procesos de transición hacia la adultez y que está notablemente influida por las estrategias familiares y por la construcción de un contexto de significados del trabajo fabril que se desarrolla desde la infancia, dada la experiencia laboral de los padres y hermanos. También lo está por la constante presencia de fábricas en el entorno cotidiano, presencia que no se limita solo a la materialidad de la construcción, sino también a los sonidos de las máquinas, los olores, los remolques entrando y saliendo, el vaivén de los obreros en los distintos turnos y su descanso en los jardines circundantes, y en los recuerdos que estas escenas se han acumulado en la biografía de estos jóvenes. 
La transición escuela-trabajo no puede ser analizada exclusivamente en la relación diádica mercado de trabajo-oferta educativa, sino que está mediada por procesos intersubjetivos de dotación de sentido a partir de la experiencia laboral familiar, las redes sociales, en particular las vecinales y familiares, y la utilización del espacio.

Las transiciones escolar, laboral y de paternidad/maternidad se desarrollan de manera casi sincrónica, aunque con vivencias significativas totalmente diferenciadas por el género. Para los varones hay una claridad en la línea de vida que hay que seguir y así lo sugieren sus transiciones: escuela-trabajo-unión/paternidad. Es de esperarse que los jóvenes trabajen a una edad menor que las mujeres, pero sobresale que, en el caso de los obreros, comenzar a trabajar en una fábrica es parte del ritual masculino para ser adulto, adultez que es vista sobre todo con relación a la formación de una familia y no necesariamente con la responsabilidad o con el "tomarse la vida en serio". Los anhelos profesionales son prácticamente nulos o por lo menos muy nebulosos e, influidos por el entorno familiar de origen, construyen perspectivas de vida de corto plazo, modestas y muy "realistas", sin ser necesariamente pesimistas.

Así, el proceso de transición hacia la adultez es experimentado y asumido por el joven obrero sin contradicciones ni cuestionamientos, aunque con insatisfacciones. Y si bien, bajo la reflexión retrospectiva, sí hubieran querido cambiar algunos de sus actos, no hay un reclamo permanente hacia ellos mismos, como sucede con las jóvenes obreras. Ello no ha de entenderse como conformismo, sino más bien como la construcción de un contexto de la experiencia vivida (Schütz, 1973), que es coherente con su entorno, fácil de asir y que dota de sentido a sus acciones en el marco de la vida cotidiana.

La transición de las jóvenes obreras es opuesta a la de sus contrapartes varones, pues se desarrolla en una secuencia escuela-maternidad/unión-trabajo. A diferencia de los varones, tienen expectativas profesionales más o menos claras y les preocupa mejorar su situación económica, no solo inmediata, sino a largo plazo: hacerse de un patrimonio, lograr estabilidad en el trabajo, conservar la salud, entre otros, para así superar las condiciones de sus padres obreros o con oficios.

Si los hombres abandonan o aplazan sus estudios como parte de un cálculo que privilegia el trabajo como componente de una identificación con una masculinidad imaginada que suaviza las nuevas responsabilidades de su paternidad, debido a que al nacimiento de su primogénito ya contaban con una trayectoria laboral incipiente, en el caso de las mujeres, el embarazo y su unión conyugal se presentan de manera abrupta, trastocando sus proyectos educativos y laborales. Así, el trabajo fabril es vivido como un fracaso, pues tuvieron que abandonar sus estudios y otros empleos que sí habían buscado (comercio y servicios). La construcción de este contexto de experiencia vivida marca toda su trayectoria de trabajo.

Por ello, las experiencias de transición de la escuela al trabajo requieren de un énfasis particular en los componentes de género, pues aún prevalece una gran inequidad en las condiciones de permanencia escolar e ingreso laboral entre los hombres y mujeres jóvenes, siendo estas las más afectadas por un mercado de trabajo cada vez más flexible y precario.

Por último, es importante destacar algunas limitaciones del estudio. Una primera limitación está dada por el análisis de trayectorias laborales apenas en curso, por lo que sería interesante, en otro momento, dar un seguimiento a los actores para conocer cómo se van desarrollando sus experiencias laborales. Otra limitación es la circunscripción del caso solo a Azcapotzalco. Contrastar si estas transiciones obreras también se dan en otros espacios manufactureros similares, como la zona maquila al norte de México u otros polígonos industriales de América Latina, daría más riqueza a la investigación sobre el tema. 


\section{Referencias}

Álvarez-Gayou, J. L. (2005). Cómo hacer investigación cualitativa: fundamentos y metodología. México, DF, México: Paidós.

Berger, P. L. \& Luckmann, T. (1997). La construcción social de la realidad. Buenos Aires, Argentina: Amorrortu.

Colín, Y. \& Villagómez, P. (2010). Evolución de la maternidad adolescente en México, 1974-2009. En Consejo Nacional de Población (Ed.), Situación de la población en México (pp. 37-54). México, DF, México: Editor.

Consejo Nacional de Población (2010). La situación actual de los jóvenes en México. México, DF, México: Autor.

Da Rosa, S., Chalfin, M., Baasch, D. \& Soares J. (2011). Sentidos y significados del trabajo: un análisis con base en diferentes perspectivas teórico-epistemológicas en psicología. Universitas Psychologica, 10, 175-188.

De la Garza, E. (2001). La formación socioeconómica neoliberal: debates teóricos acerca de la reestructuración de la producción y evidencia empírica para América Latina. México, DF, México: Plaza y Valdés.

Garabito, G. (2009). Sentido del trabajo e identificación en los jóvenes trabajadores de McDonald's. Iztapalapa, 66, 69-80.

García, M., Marino, R. \& Casal, J. (2006). Transiciones de la escuela al trabajo tras la finalización de la enseñanza secundaria obligatoria. Sociología del Trabajo, 56, 75-100.

Gergen, K. (1996). La construcción social: emergencia y potencial. En M. Pakman (Comp.), Construcciones de la experiencia humana (pp. 139-182). Barcelona, España: Gedisa.

Guzmán, C. \& Saucedo, C. (Coord.) (2007). La voz de los estudiantes: experiencias en torno a la escuela. México, DF, México: Pomares/Universidad Nacional Autónoma de México.

Heller, Á. (1998). La sociología de la vida cotidiana. Barcelona, España: Península.

Horbath, J. E. (2004). Primer empleo de los jóvenes en México. Papeles de Población, 42, 199-248.

Instituto Mexicano de la Juventud (2005). Encuesta Nacional de la Juventud, 2005: resultados preliminares. México, DF, México: Autor.

Jay, M. (2009). Cantos de experiencia: variaciones modernas sobre un tema universal. Buenos Aires, Argentina: Paidós.

Montero, F. (1987). Retorno a la fenomenología. Barcelona, España: Anthropos.

Nieto, R. (1997). Ciudad, cultura y clase obrera: una aproximación antropológica. México, DF, México: Consejo Nacional Para las Culturas y las Artes/Universidad Autónoma Metropolitana.

Oliveira, O. (2011). El trabajo juvenil en México a inicios del siglo XX. En E. Pacheco, E. De la Garza \& L. Reygadas, Trabajos atípicos y precarización del empleo (pp. 199-267). México, DF, México: El Colegio de México.

Organización Internacional del Trabajo (2006). Tendencias mundiales del empleo juvenil. Genève, Suiza: Autor.

Pérez, J. A. (2010). Las transformaciones en las edades sociales: escuela y mercados de trabajo. En R. Reguillo (Coord.), Los jóvenes en México (pp. 52-89). México, DF, México: Fondo de Cultura Económica.

Pérez, J. A. \& Urteaga, M. (2001). Los nuevos guerreros del mercado: trayectorias laborales de jóvenes buscadores de empleo. En E. Pieck (Coord.), Los jóvenes y el trabajo: la educación frente a la exclusión social (pp. 333-354). México, DF, México: Universidad Iberoamericana.

Román, J. A. \& Avendaño, C. (2002). Metáforas para re-pensar al sujeto en la sociedad del empleo: voces desde Barcelona. Psykhe, 11(2), 181-196.

Ruiz, J. I. (1996). Metodología de investigación cualitativa. Bilbao, España: Universidad de Deusto.

Saraví, G. A. (2009). Transiciones vulnerables: juventud, desigualdad y exclusión en México. México, DF, México: Centro de Investigaciones de Estudios Superiores en Antropología Social.

Schütz, A. (1973). Fenomenología del mundo social: introducción a la sociología comprensiva. Buenos Aires, Argentina: Paidós.

Vendrell, J. (2002). Masculinidades juveniles. En A. Náteras, Jóvenes, culturas e identidades urbanas (pp. 31-52). México, DF, México: Porrúa/Universidad Autónoma Metropolitana.

Fecha de recepción: Abril de 2012.

Fecha de aceptación: Agosto de 2012. 